\title{
28 Research Square \\ The Role of Virtual Consultations in Plastic Surgery During COVID-19
}

Vikram Sinha ( $\nabla$ vikramsinha@icloud.com )

king's College London https://orcid.org/0000-0002-2954-3952

Mohsan Malik

Nora Nugent

Paul Drake

Naveen Cavale

Research Article

Keywords: Plastic surgery, Telemedicine, Virtual consultation, Patient safety, Technology

Posted Date: July 23rd, 2020

DOI: https://doi.org/10.21203/rs.3.rs-45498/v1

License: (1) This work is licensed under a Creative Commons Attribution 4.0 International License.

Read Full License 


\section{Abstract}

Background: COVID-19 has led to government enforced 'lockdown' in the UK severely limiting face-to-face patient interaction. Virtual consultations present a means for continued patient access to healthcare. Our aim was to evaluate the use of virtual consultations (VCons) during lockdown and their possible role in the future.

Methods: An anonymous survey was disseminated to UK and European plastic surgeons via social media, email sharing and, via the EASAPS (European Association of Societies of Aesthetic Plastic Surgery) newsletter. Uptake of VCons, modality, effectiveness, safety and future utility were assessed.

Results: 43 senior plastic surgeons responded to the survey. The majority of the respondents $(97.7 \%)$ reported using VCons during COVID-19 lockdown, of which, 74.4\% had no prior experience. Two thirds of surgeons utilised commercial platforms such as zoom, facetime and skype. $38.1 \%$ of respondents did not know about or were unsure about adequate encryption for healthcare use, and just under a half (47.6\%) reported they were unaware of or lacking GDPR compliance. $97.6 \%$ say they are likely to use virtual consultations after lockdown.

Conclusion: Virtual consultation have had a crucial role in patient care during UK lockdown. It is clear that they will serve as an adjunct to face-to-face consultation in the future. Further regulation is required to ensure platforms offer adequate safety and security measures and are compliant with relevant data protection laws.

\section{Introduction}

The rapid spread of novel coronavirus (COVID-19), first identified in Wuhan province in December 2019, has led to a global pandemic and unprecedented challenges for healthcare institutions across the world 1 . In an attempt to contain the spread of COVID-19, the UK government implemented national curfew ("lockdown") policy on the $23^{\text {rd }}$ March 2020, to minimise community transmission. This has resulted in reduction of clinical activity across specialities demanding great flexibility from clinicians to reduce patient footfall whilst still prioritising emergencies. Face-to-face consultations were significantly reduced and outside of urgent or trauma settings and in many areas of elective practice, placed on hold entirely in an attempt to reduce the spread of disease between doctor and patient, resulting in significant restrictions to outpatient care ${ }^{1}$. Similar to allied medical specialities, plastic surgeons have made use of virtual consultations (VCons) to provide continued access to medical care whilst maintaining the rules of government lockdown ${ }^{2}$. VCons have been used to consult new patients and to reduce anticipated strain upon resumption of normal services by allowing surgeons to manage patients remotely ${ }^{3}$.

VCons are a form of telemedicine, a term used to define any medical activity involving an element of distance. Telemedicine, has been widely used in specialties such as general practice allowing patients to 
have a consultation with a doctor or nurse via telephone call without the need to see a clinician physically ${ }^{4}$. Refinement of existing video communication technology and a surge in broadband speeds mean that video communications are now more accessible, allowing patients to be examined virtually in some clinical situations. This has expanded the role of VCons into a potential tool to assess and consent patients pre-procedure and to follow up and discharge patients where necessary ${ }^{3}$. The efficiency and cost-effectiveness of VCons have been demonstrated in UK-based randomised control trials ${ }^{5}$. Furthermore, the development of purpose-built apps for VCons such as Babylon ${ }^{\circledR}$ have certified their use as secure and valid means of communicating with patients through their compliance with EU GDPR (General Data Protection Regulation) and HIPAA (Health Insurance Portability and Accountability Act) ${ }^{6,7}$.

Indeed, VCons have proven an effective tool for plastic surgeons during the COVID-19 lockdown. However, their role in future plastic surgery without or with reduced government-imposed social distancing measures is still evolving. The aim of this study was to evaluate the safety and utility of VCons during the COVID-19 lockdown, to consider its use in future practice, and, to provide guidance on safely and securely utilising and selecting a platform for VCons.

\section{Methods}

Survey

The survey consisted of an anonymous google form (supplementary) and was disseminated to UK and European plastic surgeons. Face validity was performed by senior authors (NN, PD, NC), and further construct validity was performed on the first 10 responses to assess quality of data capture.

Dissemination

The survey was disseminated via social media, email sharing and, via the EASAPS (European Association of Societies of Aesthetic Plastic Surgery) newsletter. The survey collected Reponses for 2 weeks. Data was gathered and analysed using Google forms, google drive and, google sheets.

\section{Results}

Demographics: 43 plastic surgeons responded to the survey, with mixed level of experience (fig 1), with aesthetic (69.8\%), breast (58.1\%) and skin cancer (44.2\%) subspecialties most represented. The vast majority of the respondents (97.7\%) reported using virtual consultations during COVID-19 lockdown, of which, $74.4 \%$ had no prior experience.

Modality: $72.1 \%$ of the respondents used combination of telephone and video modalities for VCons, whereas web messaging and photograph telemedicine was rarely used (fig 2). Only one third of doctors used purpose built platforms, the most common being doxy.me. The remainder opted for common commercial platforms used such as zoom, facetime and skype (fig 3). 
Consultation skills: Although, $88.1 \%$ of doctors were confident with their virtual consultation skills. $50 \%$ claimed their communication skills were the same as face-to-face, and approximately third (35.7\%) stated their communication was better in face-to-face. The majority (69\%) were not confident undertaking remote examinations, and did not utilise virtual examination. 19\% consultants felt they would list the patient for surgery following virtual consultation, in contrast to $54.8 \%$ who would prefer to review the patient for face-to-face consultation prior further management (fig 4).

Safety: $71.4 \%$ reported no concerns of personal safety during VCons. however, half reported concerns for professional safety and did not have or were unaware of medical indemnity for VCons. We found $38.1 \%$ of the respondents did not know about or were unsure about adequate encryption for healthcare use, and just under a half (47.6\%) reported they were unaware of or lacking GDPR compliance (fig 5).

Future utility: $97.6 \%$ say they are likely to use VCons after lockdown. $78 \%$ would prefer VCons to face-toface in certain situations, such as, an initial consultation or expression of interest. Those not using VCons stated that clinical concerns, safety and security online and, legal concerns were there main reasons for not using VCons. Doctors stated peer or organisation pressure may convince them to start using VCons.

\section{Discussion}

Our survey of plastic surgeons shows that the use of virtual consultations has been accelerated by COVID-19 lockdown with majority suggesting that they will continue to have a role alongside traditional face-to-face consultations in the future. However, as with any new technology, further guidance is required to ascertain the following: which platforms can be legally used, is communication sufficient to consent and discharge patients and which situations still require face-to-face consultations.

Only $32.7 \%$ of doctors were using purpose-built platforms, with the majority opting for commercial platforms such as Zoom, Skype and, Facetime. Purpose-built platforms ensure adequate encryption to prevent any unwanted intrusion of confidential information and are compliant with UK and EU digital information law ${ }^{6,8}$. During the pandemic, emergency legislation and organisational guidelines were relaxed to allow platforms to be used to facilitate patient care. However, we anticipate future guidance to allow VCons over purpose-built platforms only to ensure safety and security of both doctor and patient. $41.5 \%$ of doctors in our survey were unaware of their indemnity status whilst utilising VCons. Currently, the UK General Medical Council (GMC) has not provided specific advice regarding indemnity cover for VCons. However doctors are required to have adequate indemnity in place for all areas of their practice ${ }^{9}$.

Adequate communication during VCons is essential for a plastic surgeon in order to inform and obtain adequate consent prior to later procedures. History-taking and interviewing patients is a key skill that surgeons develop at medical school and refine during their entire career, however, during VCons verbal and non-verbal communication can be limited by technical and logistical constraints. Certain platforms can compress video making facial expressions difficult to read and hindering non-verbal communication, in telephone consultations non-verbal communication is lost entirely. Video-based consultations may 
also suffer from lag resulting in audio and video becoming unsynchronised ${ }^{10}$. However, other studies have shown that the presence of lag lead to better communication as the delay forced participants to consider their words with more thought and make greater use of turn-taking ${ }^{11}$. For procedures with fewer risks to communicate, VCons may be sufficient to consent and discharge, however, as our survey showed, the majority of surgeons (53.7\%) would still prefer a face-to-face consultation before undertaking a procedure particularly those which are more invasive. Based on our survey, we decided to formulate a checklist in order to aid clinicians in selecting a platform suitable for VCons (Table 1).

Lengthier and more invasive procedures have more risks to communicate to the patient, the surgeon has a duty when consenting to make sure the patient can understand, retain and, weigh up the information given to them during consultation. Currently, there are no laws or guidelines describing comprehensively which procedures can be consented to virtually and which procedures require a patient to be seen in person. $58.5 \%$ of doctors we surveyed state that they believe VCons can be used instead of face to face for some treatments but not all, however, the decision is currently at the surgeon's discretion. Examining a patient remotely can be problematic, $68.3 \%$ of our survey did not carry out remote examinations and only $7.3 \%$ made constant use of chaperones. Some describe tasking their patients to self-assess pre-operation such as for lymphadenopathy in lieu of a conventional examination, however, lack of examiner skill may mask any significant pathology ${ }^{12}$.

\section{Conclusion}

Virtual consultations have proved to be both time and financially efficient and have allowed patients continued access to care during this period of social distancing. It is very likely that plastic surgeons will continue utilising VCons in the future as an adjunct to traditional face-to-face consultations. Currently, the laws are relaxed upon which platforms are permitted for healthcare use, however, we believe that GDPR and HIPAA compliant purpose-built platforms should be gold standard for future practise. VCons are currently not in a state to completely replace face-to-face for all procedures, however, guidance would be helpful in the future by our professional associations as VCons become more widely utilised and their advantages and limitations become more apparent.

\section{Declarations}

\section{Compliance with Ethical Standards}

Results were collected and anonymised the participants acknowledged and provided consent for participation and publishing.

\section{Conflicts of Interest}

The authors declare that they have no conflicts of interest to disclose 


\section{Statement of human and animal rights, or ethical approval}

This article does not contain any studies with human participants or animals performed by any of the authors

\section{Informed Consent}

For this type of study consent is not required

\section{References}

1. Armstrong A, Jeevaratnam J, Murphy G, et al. A plastic surgery service response to COVID-19 in one of the largest teaching hospitals in Europe. J Plast Reconstr Aesthet Surg. 2020;73(6):1174-1205.

2. Pignatti M, Pinto V, Miralles MEL, Giorgini FA, Cannamela G, Cipriani R. How the COVID-19 pandemic changed the Plastic Surgery activity in a regional referral center in Northern Italy. $J$ Plast Reconstr Aesthet Surg. 2020.

3. Kang S, Thomas PBM, Sim DA, Parker RT, Daniel C, Uddin JM. Oculoplastic video-based telemedicine consultations: Covid-19 and beyond. Eye (Lond). 2020;34(7):1193-1195.

4. Kjaer NK, Karlsen K. [Telemedicine and general practice-future or present. Telemedicine, a way to strengthen the gatekeeper role?]. Ugeskr Laeger. 2002;164(45):5262-5266.

5. Wootton R. Telemedicine. Bmj. 2001;323(7312):557-560.

6. Phillips M. International data-sharing norms: from the OECD to the General Data Protection Regulation (GDPR). Human genetics. 2018;137(8):575-582.

7. lacobucci G. Covid-19: UK lockdown is "crucial" to saving lives, say doctors and scientists. In: British Medical Journal Publishing Group; 2020.

8. Doxy.me. Is Doxy.me HIPAA Compliant. https://help.doxy.me/en/articles/95854-is-doxy-me-hipaacompliant. Published 2020. Accessed 28th June, 2020.

9. Union TMD. Conducting Remote Consultations. https://www.themdu.com/guidance-andadvice/guides/conducting-remote-consultations. Published 2020. Accessed 28th June, 2020.

10. Greenhalgh T, Vijayaraghavan S, Wherton J, et al. Virtual online consultations: advantages and limitations (VOCAL) study. BMJ Open. 2016;6(1):e009388.

11. Krout RE, Baker FA, Muhlberger R. Designing, piloting, and evaluating an on-line collaborative songwriting environment and protocol using Skype telecommunication technology: perceptions of music therapy student participants. Music Therapy Perspectives. 2010;28(1):79-85.

12. Senie RT, Rosen PP, Lesser ML, Kinne DW. Breast self-examination and medical examination related to breast cancer stage. Am J Public Health. 1981;71(6):583-590. 


\section{Tables}

\section{Table 1. Recommendations for successful Virtual consultation}

\begin{tabular}{|l|l|}
\hline System & Recommendation \\
\hline Environment & $\begin{array}{l}\text { Accessible and compatible with a variety of devices and operating systems } \\
\end{array}$ \\
\hline Interaction & $\square$ Quilise minimal device storage and bandwidth \\
\hline Communication & $\square$ Two-way exchange of information between patient and clinician \\
& $\begin{array}{l}\text { Multi-modality consultations involving; text, audio and video. } \\
\text { Option for virtual chaperone or translator }\end{array}$ \\
\hline Integration & $\square$ Realtime communication \\
\hline Regulatory compliance & $\square$ Video communication facility to allow non verbal communication and clinical examination. \\
\hline Information Governance (GDPR and HIPAA compliant) \\
\hline Integration to patients' medical records \\
\hline
\end{tabular}

Notes: Additional items for vcons in NHS care

$\square$ Integration of referrals to and from different NHS departments (e-consults)

$\square$ NHS Data Co-ordination Board standards: Clinical Risk Management (DCB 123 and 160)

\section{Figures}




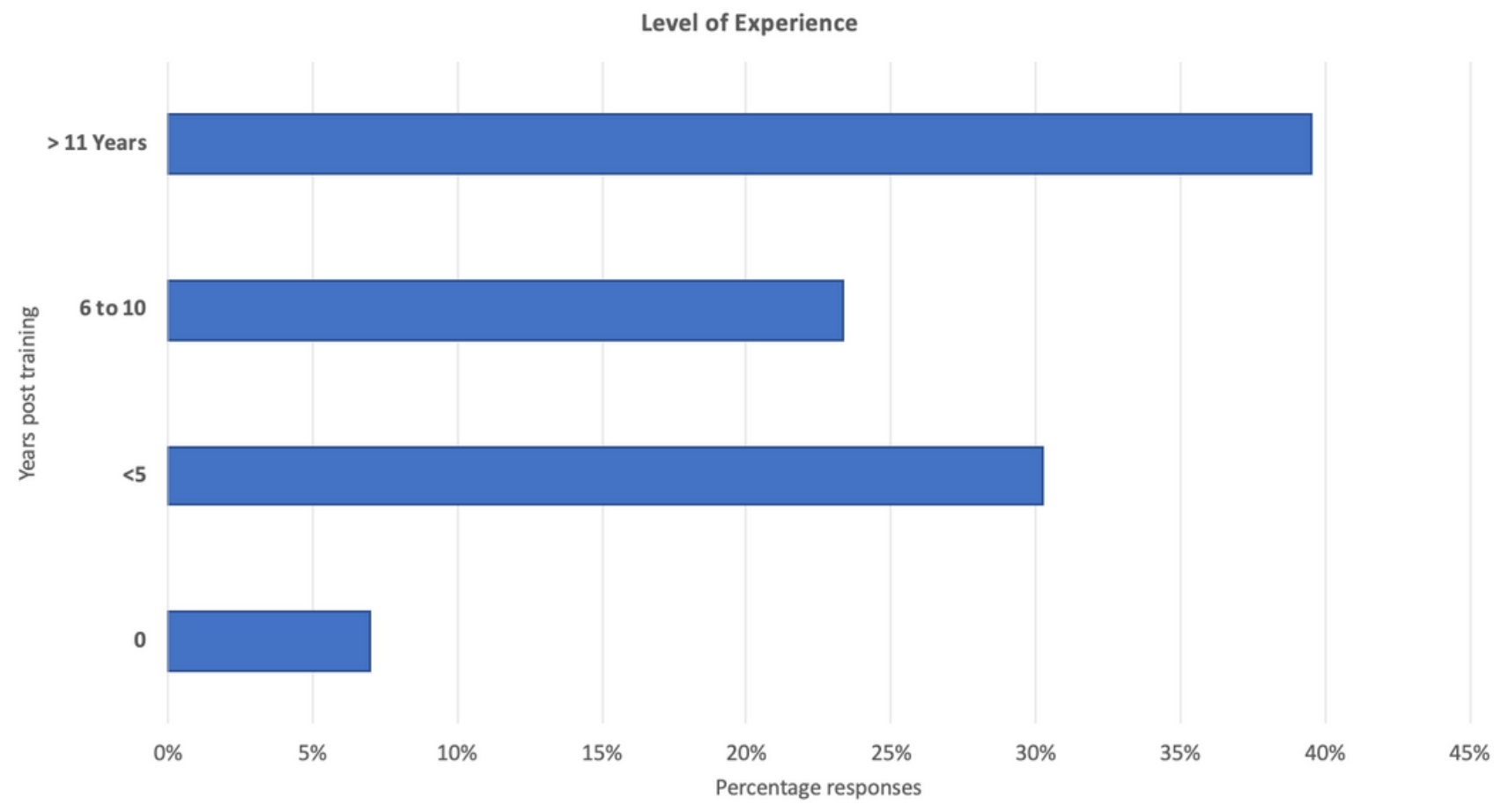

Figure 1

Range of reported experience amongst survey participants

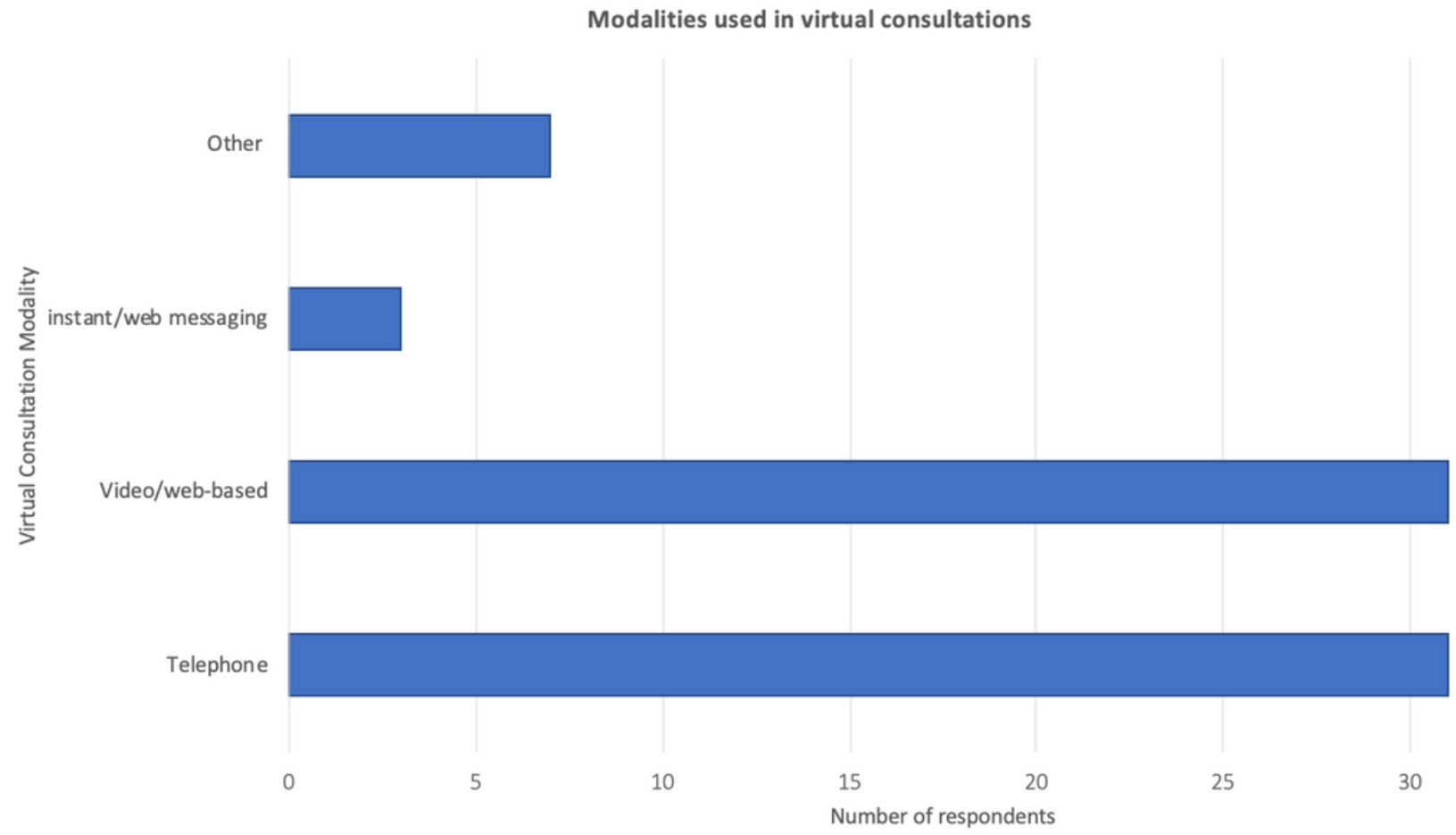

Figure 2 
Virtual consultations modalities used amongst survey participants

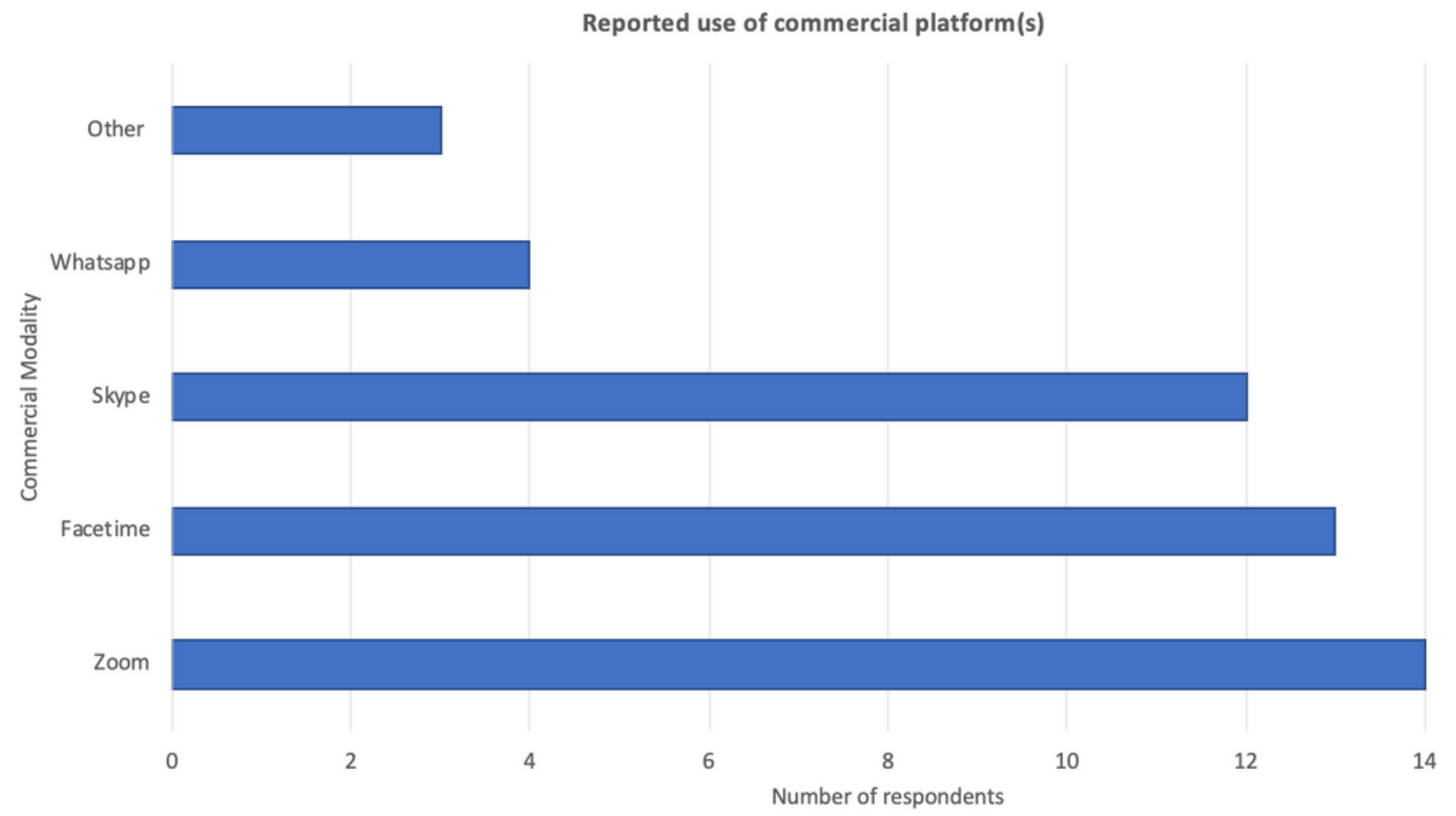

\section{Figure 3}

Reported use of commercial platform amongst survey participants

Decicison to list patients for surgical procedures following virtual consultations

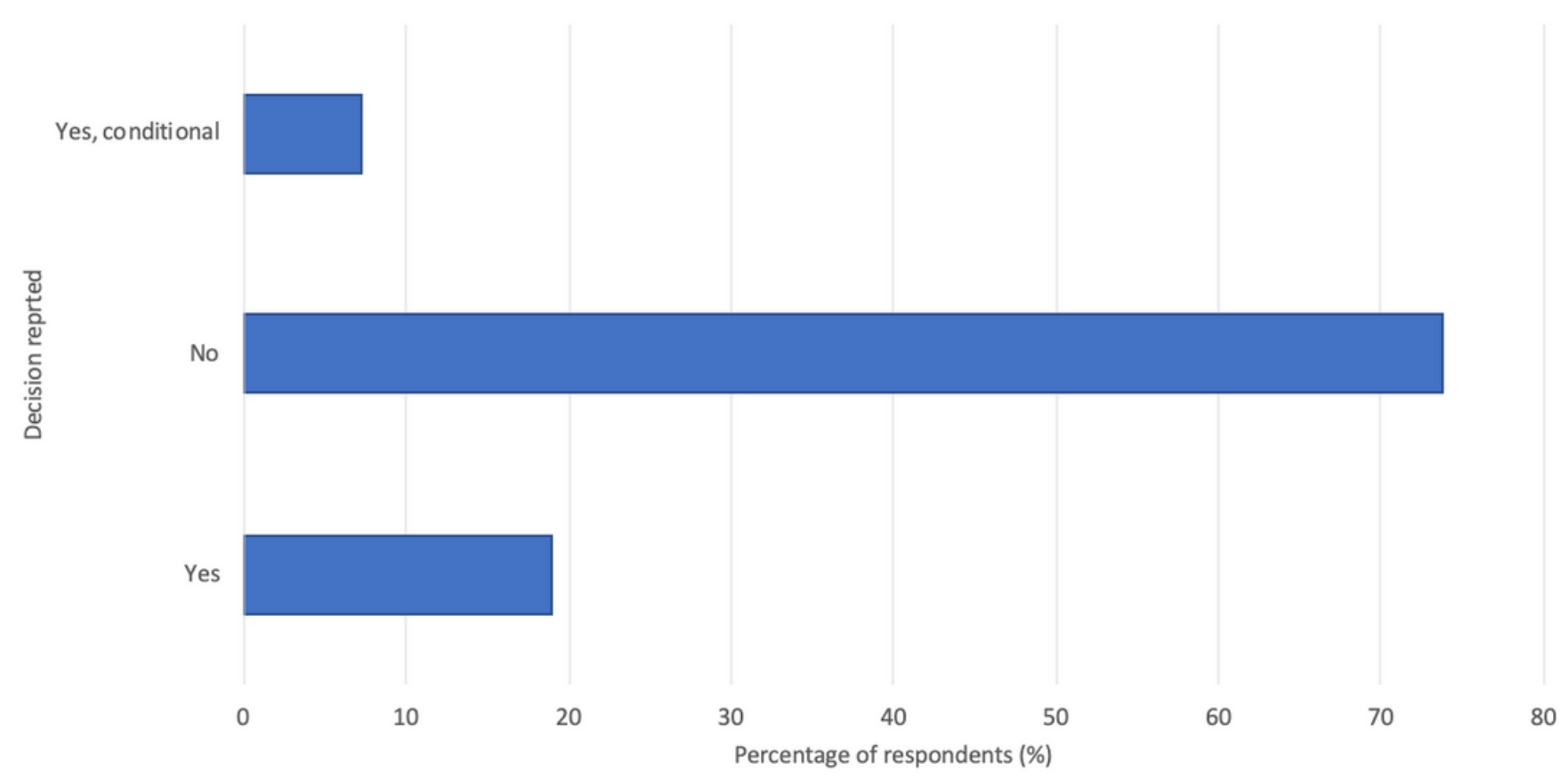

Figure 4 
Percentage of respondents that reported listing patients for surgery after virtual consultations.

Reported Safety and Security Concerns with Virtual Consultations

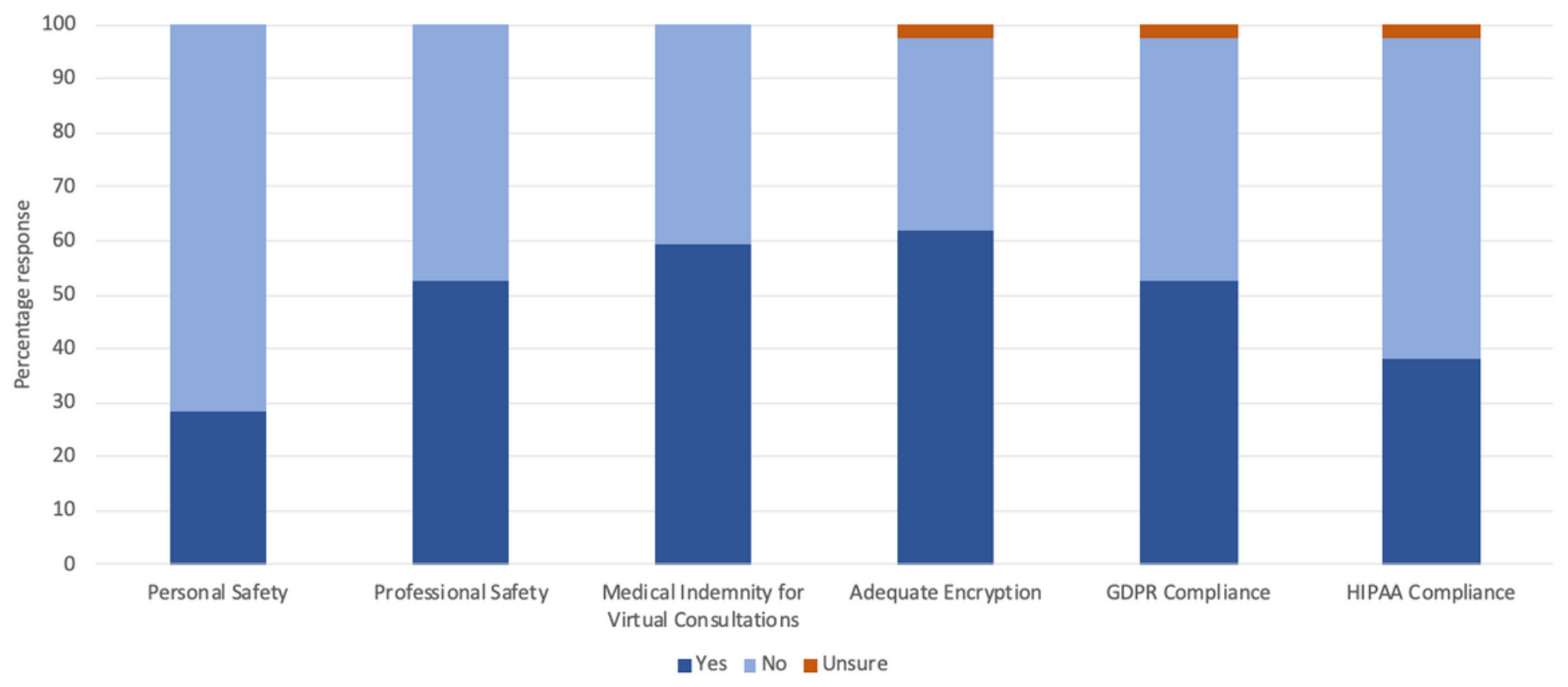

Figure 5

Reported Safety and security concerns with virtual consultation

\section{Supplementary Files}

This is a list of supplementary files associated with this preprint. Click to download.

- VirtualConsultationsInPlasticSurgeryForm.pdf 